\title{
Interactions Between Donor Age and 12-Month Estimated Glomerular Filtration Rate on Allograft and Patient Outcomes After Kidney Transplantation
}

\begin{abstract}
Wai H. Lim ${ }^{1,2 *}$, Esther Ooi ${ }^{2,3}$, Helen L. Pilmore ${ }^{4,5}$, David W. Johnson ${ }^{6,7,8}$, Stephen P. McDonald ${ }^{9,10,11,12}$, Philip Clayton ${ }^{9,10,11,12}$, Carmel Hawley ${ }^{6,7,8}$, William R. Mulley ${ }^{13,14}$, Ross Francis ${ }^{6,7}$, Michael G. Collins ${ }^{3,4}$, Bryon Jaques ${ }^{15}$, Nicholas G. Larkins ${ }^{2,16}$, Christopher E. Davies ${ }^{9,11,12}$, Kate Wyburn ${ }^{17,18}$, Steve J. Chadban ${ }^{17,18}$ and Germaine Wong ${ }^{17,19,20}$

${ }^{1}$ Department of Renal Medicine, Sir Charles Gairdner Hospital, Perth, WA, Australia, ${ }^{2}$ Medical School, University of Western Australia, Perth, WA, Australia, ${ }^{3}$ School of Biomedical Sciences, University of Western Australia, Perth, WA, Australia, ${ }^{4}$ Department of Renal Medicine, Auckland City Hospital, Auckland, New Zealand, ${ }^{5}$ Department of Medicine, University of Auckland, Auckland, New Zealand, ${ }^{6}$ Metro South Integrated Nephrology and Transplant Services, Princess Alexandra Hospital, Woolloongabba, QLD, Australia, ${ }^{7}$ Faculty of Medicine, University of Queensland, St Lucia, QLD, Australia, ${ }^{8}$ Translational Research Institute, Brisbane, QLD, Australia, ${ }^{9}$ Australia and New Zealand Dialysis and Transplant Registry, Adelaide, SA, Australia, ${ }^{10} \mathrm{Central}$ and Northern Adelaide Renal and Transplantation Services, Adelaide, SA, Australia, ${ }^{11}$ South Australia Health and Medical Research Institute, Adelaide, SA, Australia, ${ }^{12}$ Adelaide Medical School, University of Adelaide, Adelaide, SA, Australia, ${ }^{13}$ Department of Nephrology, Monash Medical Centre, Melbourne, VIC, Australia, ${ }^{14}$ Department of Medicine, Monash University, Melbourne, VIC, Australia, ${ }^{15}$ Western Australia Liver and Kidney Transplant Service, Sir Charles Gairdner Hospital, Perth, WA, Australia, ${ }^{16}$ Department of Nephrology, Perth Children's Hospital, Perth, WA, Australia,

${ }^{17}$ Faculty of Medicine and Health, University of Sydney, Sydney, NSW, Australia, ${ }^{18}$ Renal Medicine, Royal Prince Alfred Hospital, Sydney, NSW, Australia, ${ }^{19}$ Centre for Kidney Research, The Children's Hospital at Westmead, Sydney, NSW, Australia, ${ }^{20}$ Department of Renal Medicine and National Pancreas Transplant Unit, Westmead Hospital, Sydney, NSW, Australia
\end{abstract}

Reduced estimated glomerular filtration rate (eGFR) at 12-months after kidney transplantation

OPEN ACCESS

${ }^{*}$ Correspondence:

Wai H. Lim

wai.lim@health.wa.gov.au

Received: 08 November 2021 Accepted: 12 January 2022 Published: 07 February 2022

Citation:

Lim WH, Ooi E, Pilmore HL,

Johnson DW, McDonald SP,

Clayton P, Hawley C, Mulley WR,

Francis $R$, Collins $M G$, Jaques $B$,

Larkins NG, Davies CE, Wyburn K,

Chadban SJ and Wong G (2022) Interactions Between Donor Age and

12-Month Estimated Glomerular

Filtration Rate on Allograft and Patient

Outcomes After

Kidney Transplantation.

Transpl Int 35:10199.

doi: 10.3389/ti.2022.10199 is associated with increased risk of allograft loss, but it is uncertain whether donor age and types modify this relationship. Using Australia and New Zealand registry data, multivariable Cox proportional modelling was used to examine the interactive effects between donor age, types and 12-month eGFR on overall allograft loss. We included 11,095 recipients $(4,423$ received live-donors). Recipients with lowest 12-month eGFR $\left(<30 \mathrm{ml} / \mathrm{min} / 1.73 \mathrm{~m}^{2}\right)$ experienced the greatest risk of allograft loss, with adjusted HR [95\% Cl) of 2.65 [2.38-2.95] compared to eGFR of $30-60 \mathrm{ml} / \mathrm{min} / 1.73 \mathrm{~m}^{2}$; whereas the adjusted HR for highest eGFR (>60 ml/min/ $\left.1.73 \mathrm{~m}^{2}\right)$ was 0.67 [0.62-0.74]. The association of 12-month eGFR and allograft loss was modified by donor age (but not donor types) where a higher risk of allograft loss in recipients with lower compared with higher 12-month eGFR being most pronounced in the younger donor age groups $(p<0.01)$. Recipients with eGFR $<30 \mathrm{ml} / \mathrm{min} / 1.73 \mathrm{~m}^{2} 12$-months after transplantation experienced $\geq 2.5$-fold increased risk of overall allograft loss compared to those with eGFR of $>60 \mathrm{ml} / \mathrm{min} / 1.73 \mathrm{~m}^{2}$, and the magnitude of the increased risk is most marked among recipients with younger donors. Careful deliberation of other factors including donor age when considering eGFR as a surrogate for clinical endpoints is warranted.

Keywords: kidney transplantation, registry, allograft failure, patient and graft survival, estimated glomerular filtration rate, donor age, donor type 


\section{Interactions between donor age and 12-month estimated glomerular filtration rate on allograft and patient outcomes after kidney transplantation}

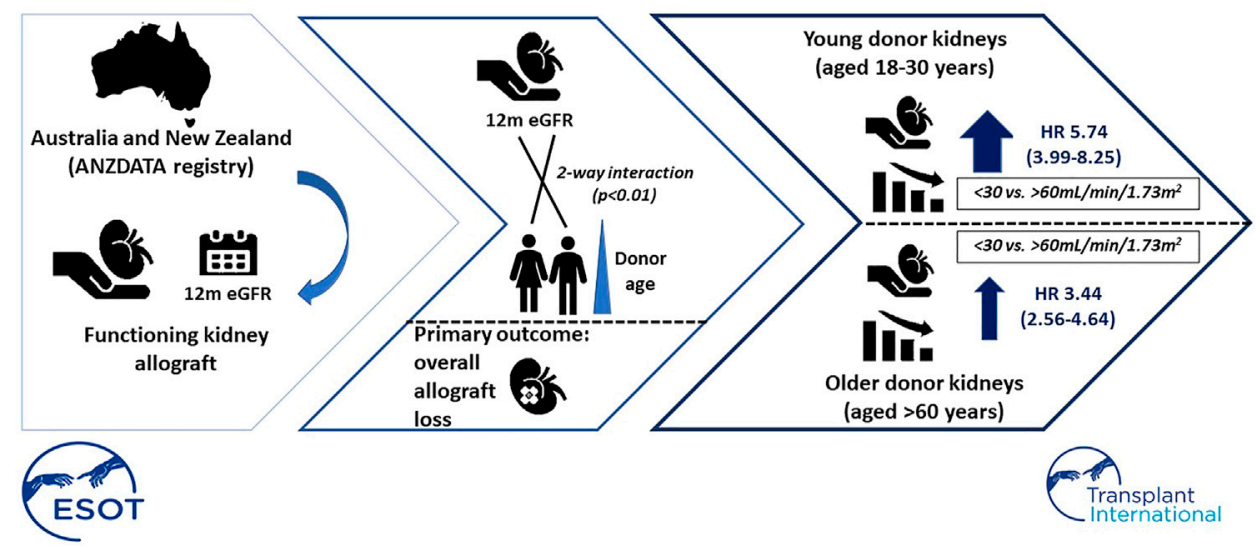

\section{INTRODUCTION}

Reduced estimated glomerular filtration rate (eGFR) is associated with an increased risk of all-cause and cardiovascular mortality in the general population and people with chronic kidney disease (1-4). There is an inverse relationship between post-transplant eGFR and the risks of adverse allograft outcomes in kidney transplantation, including death and death-censored allograft loss (5). Post-transplant kidney function, especially allograft function at 12-months post-transplant, is an important outcome measure and is considered one of the most critical outcomes for clinical trials in transplantation by patients and health professionals (5-15). In a systematic review of 169 randomized controlled trials in adult kidney transplant recipients, $60 \%$ of trials utilized creatinine-derived eGFR as a study endpoint (28\% and $61 \%$ as primary and secondary endpoints, respectively), emphasizing the clinical importance of allograft function as a potential surrogate measure of long-term allograft outcome ${ }^{7}$.

The growing use of expanded criteria (or higher Kidney Donor Profile Index [KDPI]) donors has prompted clinicians to recognize that specific donor factors, including donor age and comorbidities, may influence short- and long-term outcomes after transplantation (16-18). Many of these confounding factors have been adjusted for in the predictions for allograft loss and mortality. $(5,19,20)$ Still, no studies have explicitly examined the potential interaction between donor factors and eGFR for these outcomes. Therefore, the aim of this study was to determine whether donor age and type modify the associations between 12-month allograft function and risk of longterm allograft and patient outcomes in a contemporary cohort of kidney transplant recipients.

\section{MATERIALS AND METHODS}

\section{Study Participants}

All adult patients with kidney failure (aged 18 years or older) in Australia and New Zealand who had received first kidney transplants from adult living or deceased donors (aged 18 years or older) between 2000 and 2017 were included. Recipients of multiple organ allografts and those who had received prior transplants were excluded. Kidney transplant recipients with failed allografts within 12-months posttransplant and those without a recorded eGFR measurement at 12 months were excluded from the study. This study was approved by the University of Western Australia Human Research Ethics Committee (reference: 2019/RA/4/20/4584) and is reported here according to The Strengthening the Reporting of Observational Studies in Epidemiology (STROBE) guidelines (21).

\section{Demographics and Clinical Characteristics}

Baseline characteristics included donor factors (age, donor type [living or deceased], sex, diabetes, hypertension and smoking history); recipient factors (age, sex, ethnicity, body mass index [BMI] at 12-months post-transplant, waiting time pre-transplant [in years], prevalent comorbidities [presence of diabetes, coronary artery disease, cerebrovascular disease or peripheral vascular disease pre-transplantation], smoking history and cause of kidney failure); and transplant-related factors (peak percentage panel reactive antibody [\%PRA], number of human leukocyte antigen [HLA] A, B and DR mismatches, transplant era, place of transplantation [Australian states or New Zealand] and initial immunosuppressive agents).

\section{Exposure and Clinical Outcomes}

Post-transplant kidney function, especially allograft function at 12months post-transplant, was chosen as the exposure of interest for three reasons: 1) It is one of the most important clinical outcomes identified by both patients, caregivers and healthcare professionals $(14,15,22) ; 2)$ Previous epidemiological studies have found a strong association between 12-month allograft function and long-term survival $(5,8,11,12)$; 3$)$ There is established evidence to show 


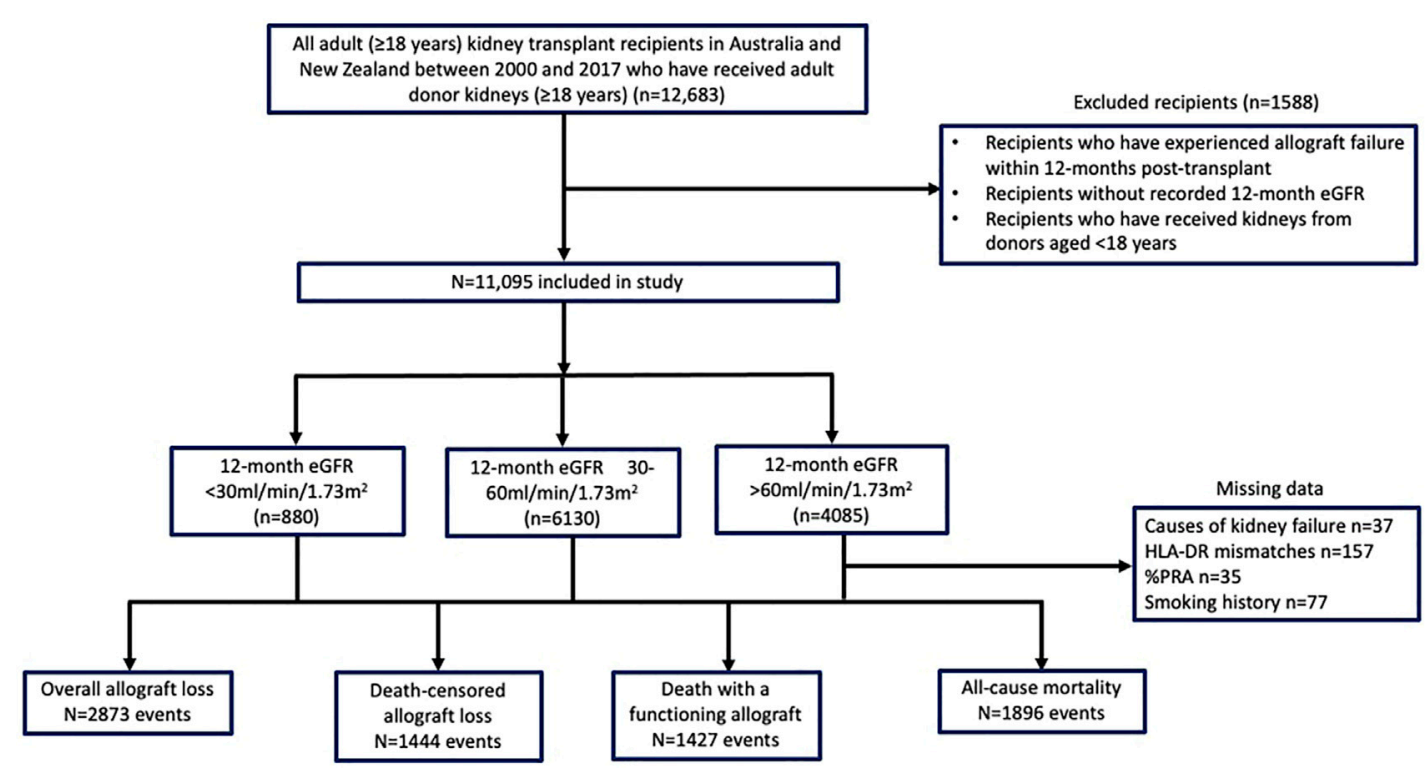

FIGURE 1 | Flow diagram of the study cohort of adult kidney transplant recipients in Australia and New Zealand between 2000 and 2017.

the effect of treatments such as belatacept, on 12-month allograft function has led to improved long-term allograft survival in kidney transplant recipients $(9,23,24)$. Recipients' 12 -month eGFR values were calculated using the Chronic Kidney Disease Epidemiology Collaboration (CKD-EPI) equation (25), and categorized into prior clinically defined thresholds of $>60,30$ to 60 and $<30 \mathrm{ml} / \mathrm{min} /$ $1.73 \mathrm{~m}^{2}$. The primary outcome of this study was overall allograft loss (includes death-censored allograft loss and death with a functioning graft). The secondary outcomes were death-censored allograft loss, death with a functioning allograft and all-cause mortality (including death after allograft loss).

\section{Statistical Analysis}

Data are presented as number (proportion), mean (standard deviation $[\mathrm{SD}]$ ) and median (interquartile range [IQR]) where appropriate, with comparisons between groups examined by chisquare test, analysis of variance (ANOVA) or Kruskal-Wallis test, respectively. The associations between 12 -month eGFR, primary and secondary outcomes were examined using adjusted Cox regression models. Grouped LASSO (least absolute shrinkage and selection operator) regularized logistic regression was used for variables selection (26). The variables of importance were HLA-DR mismatches, prior smoking history, prevalent coronary artery disease, prevalent cerebrovascular disease, prevalent diabetes, primary cause of kidney failure, dialysis duration and peak \%PRA in the models that considered overall and deathcensored allograft loss, with the addition of recipient age and recipient smoking history in the models for death with a functioning allograft and all-cause mortality. In all Cox regression models, donor age, donor types, transplantation states and transplant era were also included as covariates.

The 12-month eGFR and donor age was considered as the two-way interaction term, and 12-month eGFR, donor age and donor types were considered as the three-way interaction term. We first tested the interaction using continuous measures of 12month eGFR and donor age, with significant interactions $(p<$ 0.01 ) observed for the outcome of overall allograft loss. We next constructed models evaluating the two-way interaction between categories of 12-month eGFR (according to the prior clinically defined thresholds of $>60,30$ to 60 and $<30 \mathrm{ml} / \mathrm{min} / 1.73 \mathrm{~m}^{2}$ ) and donor age, with donor age thresholds informed by restricted cubic splines ( 5 knots; Supplementary Figure S1). There was a significant interaction $(p<0.1)$ between categories of 12 -month eGFR and donor age for overall allograft loss, but not for deathcensored allograft loss. However, a three-way interaction between 12-month eGFR, donor age and donor type were not observed for allograft and patient outcomes.

The estimates were expressed as adjusted hazard ratio (HR) and corresponding 95\% confidence intervals (95\% CI). The proportional hazard assumptions for all Cox regression models were examined graphically by Schoenfeld residuals with no evidence of departures from proportional hazards for allograft loss or mortality. A sensitivity analysis examining the associations between 12-month eGFR and outcomes were undertaken with the inclusion of other donor characteristics of diabetes, hypertension and smoking history in the Cox regression models. All analyses were undertaken using SAS (version 9.4; SAS Institute Inc., Cary, NC) and STATA (Version 15; StataCorp, College Station, TX), with $p$-values of $<0.05$ in two-tailed testing considered statistically significant.

\section{RESULTS}

Of the 12,683 first kidney transplants performed in 2000-2017, we excluded 1,588 recipients who lost their allografts within 
TABLE 1 | Baseline characteristics of kidney transplant recipients transplanted between 2000 and 2017, stratified by 12-month estimated glomerular filtration rate categories.

\begin{tabular}{|c|c|c|c|c|}
\hline & \multicolumn{3}{|c|}{ eGFR categories } & \multirow[t]{2}{*}{ p-value } \\
\hline & $\begin{array}{c}<30 \mathrm{ml} / \mathrm{min} / 1.73 \mathrm{~m} 2 \\
(\mathrm{n}=\mathbf{8 8 0})\end{array}$ & $\begin{array}{c}30-60 \mathrm{ml} / \mathrm{min} / 1.73 \mathrm{~m} 2 \\
(\mathrm{n}=6,130)\end{array}$ & $\begin{array}{c}>60 \mathrm{ml} / \mathrm{min} / 1.73 \mathrm{~m} 2 \\
(\mathrm{n}=4,085)\end{array}$ & \\
\hline \multicolumn{5}{|l|}{ Recipient characteristics } \\
\hline Age (year, mean $\pm S D$ ) & $52.4 \pm 13.1$ & $50.1 \pm 12.8$ & $46.6 \pm 13.7$ & $<0.01$ \\
\hline Female $(n, \%)$ & $351(39.9)$ & $2,145(35.0)$ & $1,561(38.2)$ & $<0.001$ \\
\hline $\mathrm{BMl}(\mathrm{kg} / \mathrm{m} 2$, mean $\pm \mathrm{SD})$ & $28.0 \pm 5.9$ & $28.2 \pm 5.2$ & $27.2 \pm 5.4$ & $<0.001$ \\
\hline Ethnicity (n, \%) & & & & $<0.001$ \\
\hline Caucasian & 668 (75.9) & $4,780(78.0)$ & $3,000(73.4)$ & \\
\hline Indigenous Australian & $38(4.3)$ & $177(2.9)$ & $96(2.4)$ & \\
\hline New Zealand Māori & $19(2.2)$ & $171(2.8)$ & $85(2.1)$ & \\
\hline Others/not recorded & $155(17.6)$ & $1,002(16.3)$ & $904(22.1)$ & \\
\hline Former/current smokers ( $\mathrm{n}, \%)$ & $434(49.9)$ & $2,749(45.1)$ & $1,632(40.3)$ & $<0.001$ \\
\hline Coronary artery disease $(n, \%)$ & $117(13.3)$ & $642(10.5)$ & $325(8.0)$ & $<0.001$ \\
\hline Peripheral vascular disease (n, \%) & $69(7.8)$ & $386(6.3)$ & $170(4.2)$ & $<0.001$ \\
\hline Cerebrovascular disease (n, \%) & $62(7.1)$ & $312(5.1)$ & $151(3.7)$ & $<0.001$ \\
\hline Diabetes ( $\mathrm{n}, \%)$ & $190(21.6)$ & $1,031(16.8)$ & $637(15.6)$ & $<0.001$ \\
\hline Cause of kidney failure (n, \%) & & & & 0.005 \\
\hline Diabetes & $130(14.8)$ & $703(11.5)$ & $424(10.4)$ & \\
\hline Glomerulonephritis & $370(42.1)$ & $2,726(44.6)$ & $1782(43.8)$ & \\
\hline Vascular & $56(6.4)$ & $376(6.1)$ & $256(6.3)$ & \\
\hline Cystic & $131(14.9)$ & $1,029(16.8)$ & $677(16.7)$ & \\
\hline Analgesic Nephropathy & $7(0.8)$ & $39(0.6)$ & $14(0.3)$ & \\
\hline Other or Unknown & $185(21.0)$ & $1,242(20.4)$ & $911(22.5)$ & \\
\hline Waiting time (years, mean \pm SD) & $3.6 \pm 2.9$ & $2.8 \pm 2.7$ & $2.5 \pm 2.5$ & $<0.001$ \\
\hline eGFR $(\mathrm{ml} / \mathrm{min} / 1.73 \mathrm{~m} 2 \text {, mean } \pm \mathrm{SD})^{\mathrm{a}}$ & $23.0 \pm 5.6$ & $46.5 \pm 8.1$ & $74.9 \pm 12.6$ & $<0.001$ \\
\hline 12-month eGFR categories $(\mathrm{n}, \%)^{\mathrm{a}}$ & & & & $<0.001$ \\
\hline$\geq 90$ & $0(0.0)$ & $0(0.0)$ & $510(12.5)$ & \\
\hline$>60-89$ & $0(0.0)$ & $0(0.0)$ & $3,575(87.5)$ & \\
\hline $45-60$ & $0(0.0)$ & $3,588(58.5)$ & $0(0.0)$ & \\
\hline $30-44$ & $0(0.0)$ & $2,542(41.5)$ & $0(0.0)$ & \\
\hline $15-29$ & 791 (89.9) & $0(0.0)$ & $0(0.0)$ & \\
\hline$<15$ & $89(10.1)$ & $0(0.0)$ & $0(0.0)$ & \\
\hline \multicolumn{5}{|l|}{ Donor characteristics } \\
\hline Age (years, mean \pm SD) & $57.1 \pm 12.5$ & $51.3 \pm 12.4$ & $42.4 \pm 13.3$ & $<0.001$ \\
\hline Female $(n, \%)$ & $450(51.8)$ & $3,126(53.1)$ & 1709 (43.5) & $<0.001$ \\
\hline Living donor $(\mathrm{n}, \%)$ & $201(22.8)$ & $2,419(39.5)$ & $1787(43.7)$ & $<0.001$ \\
\hline Deceased DCD donor (n, \%) & $123(14.0)$ & $669(10.9)$ & $369(9.0)$ & 0.126 \\
\hline Donor diabetes & $66(7.5)$ & $272(4.4)$ & $101(2.5)$ & $<0.001$ \\
\hline Donor hypertension & $312(35.5)$ & $1,290(21.0)$ & $432(10.6)$ & $<0.001$ \\
\hline Donor smoking history & $228(25.9)$ & $502(24.5)$ & $1,201(29.4)$ & $<0.001$ \\
\hline \multicolumn{5}{|l|}{ Transplant characteristics } \\
\hline HLA-ABDR mismatches (mean \pm SD) & $3.7 \pm 1.7$ & $3.4 \pm 1.7$ & $3.3 \pm 1.7$ & $<0.001$ \\
\hline Ischemic time (hours, mean \pm SD) & $10.9 \pm 6.2$ & $8.7 \pm 6.1$ & $8.1 \pm 6.0$ & $<0.001$ \\
\hline Peak percentage PRA (n, \%) & & & & $<0.001$ \\
\hline $0-10$ & $663(75.5)$ & $5,077(83.1)$ & $3,378(83.0)$ & \\
\hline $11-50$ & $140(15.9)$ & $667(10.9)$ & $463(11.4)$ & \\
\hline $51-80$ & $41(4.7)$ & $190(3.1)$ & $128(3.1)$ & \\
\hline$>80$ & $34(3.9)$ & $176(2.9)$ & $103(2.5)$ & \\
\hline Transplant year (n, \%) & & & & $<0.001$ \\
\hline 2000-2004 & $221(25.1)$ & $1,410(23.0)$ & $729(17.8)$ & \\
\hline $2005-2008$ & $154(17.5)$ & $1,236(20.2)$ & $788(19.3)$ & \\
\hline 2009-2012 & $210(23.9)$ & $1,441(23.5)$ & $1,084(26.5)$ & \\
\hline 2013-2017 & $295(33.5)$ & $2043(33.3)$ & $1,484(36.4)$ & \\
\hline Prednisolone at 12 m (n, \%) & $869(98.8)$ & $6,055(98.8)$ & $4,006(98.1)$ & 0.012 \\
\hline Calcineurin-inhibitor at $12 \mathrm{~m}(\mathrm{n}, \%)$ & & & & 0.005 \\
\hline None & $12(1.4)$ & $80(1.3)$ & $68(1.7)$ & \\
\hline Cyclosporin & $161(18.3)$ & $1,375(22.4)$ & $35(0.9)$ & \\
\hline Tacrolimus & 707 (80.3) & 4,675 (76.3) & 3,982 (97.4) & \\
\hline Anti-metabolite at $12 \mathrm{~m}(\mathrm{n}, \%)$ & & & & 0.991 \\
\hline Non & $15(1.7)$ & $100(1.6)$ & $110(6.5)$ & \\
\hline Azathioprine & $7(0.8)$ & $47(0.8)$ & $117(7.0)$ & \\
\hline Mycophenolic acid & $858(97.5)$ & $5,983(97.6)$ & $1,456(86.5)$ & \\
\hline
\end{tabular}

${ }^{a}$ One-year post-transplantation.

$L D$, live donor; $D D$, deceased donor; ESKD, end-stage kidney disease, BMI, body mass index; eGFR, estimated glomerular filtration rate by Chronic Kidney Disease Epidemiology Collaboration equation; DCD, donation after circulatory death; HLA, human leukocyte antigen; PRA, panel reactive antibody; mTOR, mammalian target of rapamycin. 
12 months post-transplant or had no recorded 12-month eGFR, leaving a study cohort of 11,095 recipients (Figure 1). The mean (SD) age of the study cohort was 49 (13) years, and 37\% were females. Eight hundred and eighty recipients (7.9\%) had 12month eGFR $<30 \mathrm{ml} / \mathrm{min} / 1.73 \mathrm{~m}^{2}$ and 4,085 (36.8\%) had eGFR $<30$ and $>60 \mathrm{ml} / \mathrm{min} / 1.73 \mathrm{~m}^{2}$.

Table 1 shows the baseline characteristics of the study cohort, stratified by 12-month eGFR thresholds. Recipients with 12month eGFR of $>60 \mathrm{ml} / \mathrm{min} / 1.73 \mathrm{~m}^{2}$ were younger, less likely to have prevalent vascular disease or diabetes, and had shorter mean waiting time than recipients with 12 -month eGFR $\leq 60 \mathrm{ml} /$ $\mathrm{min} / 1.73 \mathrm{~m}^{2}$. Recipients with 12 -month eGFR values of $>60 \mathrm{ml} /$ $\mathrm{min} / 1.73 \mathrm{~m}^{2}$ were more likely to have received living donor kidneys and of younger donor age compared to those with 12month eGFR of $\leq 60 \mathrm{ml} / \mathrm{min} / 1.73 \mathrm{~m}^{2}$. The proportion of kidney transplant recipients with 12-month eGFR $>60 \mathrm{ml} / \mathrm{min} / 1.73 \mathrm{~m}^{2}$ increased from $30.9 \%$ between 2000 and 2004 to $38.8 \%$ between 2013 and 2017. Conversely, the proportion of recipients with 12month eGFR $<30 \mathrm{ml} / \mathrm{min} / 1.73 \mathrm{~m}^{2}$ reduced from $9.4 \%$ between 2000 and 2004 to $7.7 \%$ between 2013 and 2017 .

Donor Age Categories and 12-Month eGFR A higher proportion of recipients who received kidneys from younger donors aged 18-30 years had 12-month eGFR >60 ml/ $\mathrm{min} / 1.73 \mathrm{~m}^{2}$ compared to recipients of donor kidneys aged $>30-60$ and $>60$ years. Conversely, approximately $17 \%$ of recipients with older donor kidneys (aged $>60$ years) had 12month eGFR of $<30 \mathrm{ml} / \mathrm{min} / 1.73 \mathrm{~m}^{2}$ compared to $3 \%$ of recipients with younger donor kidneys (aged 18-30 years) (Figure 2 and Supplementary Table S1).

\section{Association Between 12-Month eGFR and Overall Allograft Loss}

The estimates of the main model for overall allograft loss are shown in Table 2. Compared to recipients with 12-month eGFR of 30-60 ml/ $\mathrm{min} / 1.73 \mathrm{~m}^{2}$, recipients with the lowest 12 -month eGFR $(<30 \mathrm{ml} /$ $\mathrm{min} / 1.73 \mathrm{~m}^{2}$ ) experienced the greatest risk of overall allograft loss (adjusted HR [95\% CI]: 2.65 [2.38, 2.95]); where those with the highest eGFR at 12-months experienced a lower risk of overall allograft loss (adjusted HR 0.67 [0.62-0.74]). Compared to recipients of older donor kidneys, recipients with younger donor kidneys experienced a reduced risk of overall allograft loss.

\section{Interaction Between Donor Age, 12-Month eGFR and Overall Allograft Loss}

Figure 3 shows the adjusted HRs and 95\% CI for eGFR categories and overall allograft loss stratified by donor age subgroups of $18-30,>30-60$ and $>60$ years. In recipients of kidneys from younger donors (aged 18-30 years), the adjusted HRs for overall allograft loss were highest in those with the lowest 12-month eGFR values $\left(<30 \mathrm{ml} / \mathrm{min} / 1.73 \mathrm{~m}^{2}\right.$ : HR 5.74 [95\% CI 3.99, 8.25]; $30-60 \mathrm{ml} / \mathrm{min} / 1.73 \mathrm{~m}^{2}$ : HR 1.37 [95\% CI 1.13, 1.66]; >60 ml/min/ $1.73 \mathrm{~m}^{2}$ : referent). In recipients of kidneys from older donors aged $>60$ years, the HRs for overall allograft loss were attenuated at lower 12 -month eGFR values $\left(<30 \mathrm{ml} / \mathrm{min} / 1.73 \mathrm{~m}^{2}\right.$ : HR 3.44
[95\% CI 2.56, 4.64]; 30-60 ml/min/1.73 $\mathrm{m}^{2}:$ HR 1.45 [95\% CI $1.09,1.92]$; $>60 \mathrm{ml} / \mathrm{min} / 1.73 \mathrm{~m}^{2}$ : referent) (Table 2 and Figure 3).

Figures 4A-C show the adjusted HR for overall allograft loss across the continuum of 12-month eGFR, stratified by donor age groups. The inflection points of the survival curves corresponding to an increased risk of overall allograft loss occurred at lower eGFR values for recipients of older donor kidneys than younger donor kidneys.

\section{Association Between 12-Month eGFR and Death Censored Allograft Loss, Death With a Functioning Allograft and All-Cause Mortality}

The estimates of the main models (without interaction) for death censored allograft loss, death with a functioning allograft and allcause mortality are shown in Table 2 . Compared to 12 -month eGFR of $30-60 \mathrm{ml} / \mathrm{min} / 1.73 \mathrm{~m}^{2}$, the adjusted HR for 12 -month eGFR of $<30 \mathrm{ml} / \mathrm{min} / 1.73 \mathrm{~m}^{2}$ was $3.94 \quad(3.44,4.53)$ for death-censored allograft loss, $1.30(1.09,1.54)$ for death with a functioning allograft and $1.78(1.56,2.04)$ for all-cause mortality. The respective HRs for 12-month eGFR of $>60 \mathrm{ml} / \mathrm{min} / 1.73 \mathrm{~m}^{2}$ were $0.56(0.49,0.64), 0.82(0.73,0.93)$ and $0.77(0.69,0.86)$. These relationships were not modified by donor age.

\section{Sensitivity Analysis}

A greater proportion of recipients with 12-month eGFR of $<30 \mathrm{ml} / \mathrm{min} / 1.73 \mathrm{~m}^{2}$ received kidneys from donors with a history of diabetes or hypertension compared to recipients with higher 12-month eGFR (Table 1). In the sensitivity analysis which included these additional donor characteristics (donor diabetes, donor hypertension and donor smoking history), the two-way interaction between 12-month eGFR and donor age remained statistically significant for overall allograft loss. Figure 3 shows the adjusted HRs and 95\%CI for eGFR categories and overall allograft loss according to the donor age subgroups of $18-30,>30-60$ and $>60$ years.

\section{DISCUSSION}

In this contemporary cohort of kidney transplant recipients, recipients with 12 -month eGFR less than $30 \mathrm{ml} / \mathrm{min} / 1.73 \mathrm{~m}^{2}$ experienced at least a 2.5 fold increased risk of overall allograft loss compared to those with higher eGFR at 12 month $\left(>60 \mathrm{ml} / \mathrm{min} / 1.73 \mathrm{~m}^{2}\right)$. This association was modified by donor age but not donor types. Recipients of younger donor kidneys with a lower 12 -month eGFR value of less than $30 \mathrm{ml} / \mathrm{min} / 1.73 \mathrm{~m}^{2}$ experienced up to 6 -times greater risk of overall allograft loss compared to those with higher 12-month eGFR values. This association was attenuated in recipients with older donor kidneys.

Observational data shown a direct association between donor age and kidney function at 12-months and long-term allograft 


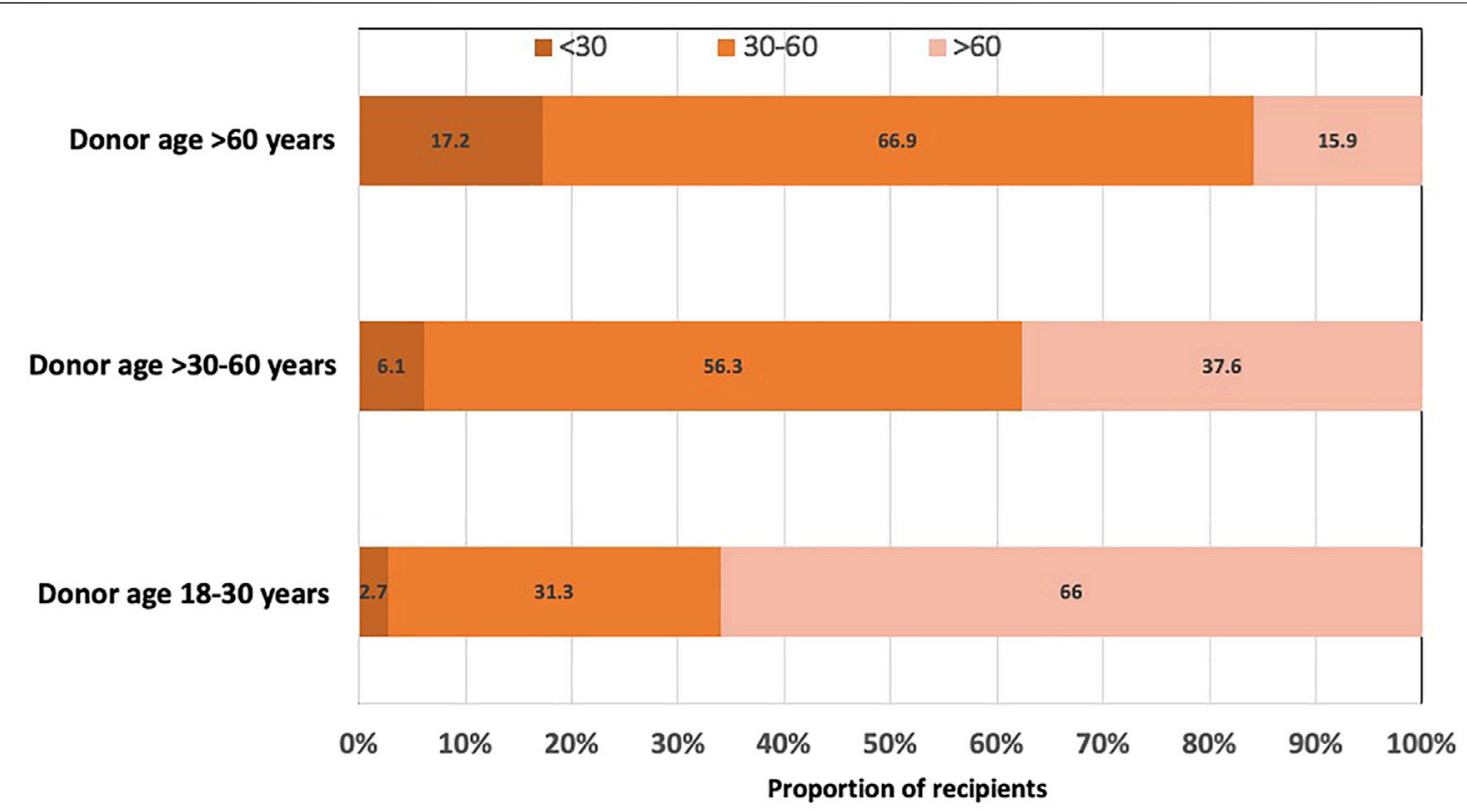

FIGURE 2 |Bar graph showing the proportion of kidney transplant recipients with 12-month estimated glomerular filtration rate (eGFR) of $<30,30-60$ and $>60$ ml/ $\mathrm{min} / 1.73 \mathrm{~m}^{2}$, stratified by donor age groups (18-30, >30-60 and $>60$ years).

and patient survivals $(5,8,20,27-29)$. but our observed interactive effects between donor age and eGFR at 12 months on allograft loss is novel. Our study findings suggest that the effects of reduced short-term allograft function at 12-month on longer term allograft outcome differs in recipients of younger and older donor kidneys, with the magnitude of the risk for overall allograft loss being higher for recipients of younger donor kidneys with lower 12-month eGFR values than those who received older donor kidneys. The inflection point for the increased risk of allograft loss occurred at a lower eGFR for older donor kidneys than younger donor kidneys. Our current findings may imply that clinical events or disease phenotypes that may have led to a reduced eGFR at 12-months for recipients with younger donor kidneys are different from recipients of older donor kidneys who had reduced eGFR at 12-months. However, these findings also suggest that donor age alone is unlikely the only contributing factor in modifying the association between 12-month eGFR and allograft outcomes. Other mechanisms or influences such as the different etiology of the allograft dysfunction (such as disease recurrence, vascular complications, cellular or antibody-mediated rejection, BK viral nephropathy), the differing susceptibility of the donor kidneys (of varying ages) to clinical insults and the presence of competing events such as death with a functioning allograft may have affected the trajectory for allograft loss for each eGFR threshold according to incremental donor age subgroups.

In a systematic review of 169 randomized controlled trials in kidney transplantation, eGFR was a primary or secondary endpoint in $60 \%$ of the trials (7). Clinical trials powered to hard clinical endpoints such as allograft survival are often not feasible in kidney transplantation. Therefore, eGFR is likely to continue to be used as a surrogate measure of allograft survival. In the two largest clinical trials ever conducted in kidney transplantation, the primary endpoint was 12-month eGFR (Efficacy Limiting Toxicity Elimination [ELITE]-Symphony study [n $=1,645]$; mean [SD] donor age 45-46 [15-16] years; published 2007) or a composite of acute rejection or eGFR of $<50 \mathrm{ml} / \mathrm{min} / 1.73 \mathrm{~m}^{2}$ at 12 -months (TRANSplant eFficacy and Safety Outcomes With an eveRolimusbased regiMen [TRANSFORM] study [n = 2037]; mean [SD] donor age of 48 [15] years, published 2018), indicating that eGFR will likely remain one of the best and practical index measures for longer-term kidney allograft outcome $(11,12)$. Consequently, a greater understanding of the limitations of the prognostic significance of a single timepoint eGFR is critical when considering clinical trial design and when interpreting the results of clinical trials in kidney transplantation.

Estimated GFR, however, does not necessarily provide accurate quantification of the amount and etiology of the "pathological" acute and chronic changes in the allograft biopsy, which can be influenced by multiple patient- and transplant-related factors, such as the primary cause of kidney failure, body size, age and post-transplant clinical events (e.g. disease recurrence, antibody mediated rejection) and therefore, kidney allograft biopsies are often required to guide clinical management (30). Our study suggests that donor age should be considered when interpreting the clinical applicability and prognostic significance of a single time point eGFR value such that the proportion of recipients attaining different 12-month eGFR thresholds and the association between eGFR and risk of overall allograft loss may be conditional on the effects of donor age. This finding also suggests the need for careful consideration when utilizing a single time point eGFR value as a surrogate measure for overall allograft loss in kidney transplant trials. 
TABLE 2 | Association between 12-month eGFR, long-term allograft and patient outcomes (main effects models).

\begin{tabular}{|c|c|c|c|c|}
\hline & $\begin{array}{l}\text { Overall allograft loss } \\
\text { (adjusted HR [95\% } \\
\text { Cl]) }\end{array}$ & $\begin{array}{l}\text { Death censored allograft } \\
\text { loss (adjusted HR } \\
\left.\left[\begin{array}{ll}95 \% & \mathrm{Cl}\end{array}\right]\right)\end{array}$ & $\begin{array}{c}\text { Death with a } \\
\text { functioning allograft (adjusted } \\
\text { HR }[95 \% \mathrm{Cl}])\end{array}$ & $\begin{array}{l}\text { All-cause mortality (adjusted } \\
\text { HR }[95 \% \mathrm{Cl}])\end{array}$ \\
\hline \multicolumn{5}{|l|}{ 12-month eGFR (mL/min/1.73m2) } \\
\hline$<30$ & $2.65(2.38,2.95)$ & $3.94(3.44,4.53)$ & $1.30(1.09,1.54)$ & $1.78(1.56,2.04)$ \\
\hline $30-60$ & 1.00 & 1.00 & 1.00 & 1.00 \\
\hline$>60$ & $0.67(0.62,0.74)$ & $0.56(0.49,0.64)$ & $0.82(0.73,0.93)$ & $0.77(0.69,0.86)$ \\
\hline \multicolumn{5}{|l|}{ Donor factors } \\
\hline Live donor (ref: deceased donor) & $0.92(0.84,1.01)$ & $0.81(0.72,0.91)$ & $0.91(0.79,1.05)$ & $0.90(0.80,1.01)$ \\
\hline \multicolumn{5}{|l|}{ Donor age (years) } \\
\hline $18-30$ & $0.79(0.69,0.90)$ & $0.69(0.57,0.85)$ & $0.92(0.77,1.11)$ & $0.87(0.74,1.02)$ \\
\hline$>30-60$ & $0.88(0.80,0.97)$ & $0.90(0.79,1.04)$ & $0.90(0.79,1.03)$ & $0.90(0.80,1.02)$ \\
\hline$>60$ & 1.00 & 1.00 & 1.00 & 1.00 \\
\hline \multicolumn{5}{|l|}{ Recipient factors } \\
\hline Recipient age (in years) & - & $0.96(0.95,0.96)$ & $1.07(1.06,1.11)$ & $1.06(1.05,1.06)$ \\
\hline Prior smoking history (ref: non-smoker) & $1.30(1.20,1.40)$ & - & $1.35(1.22,1.50)$ & $1.37(1.25,1.50)$ \\
\hline Prior coronary artery disease & $1.30(1.18,1.44)$ & $1.74(1.51,2.01)$ & $1.03(0.90,1.18)$ & $1.21(1.08,1.36)$ \\
\hline Prior cerebrovascular disease & $1.42(1.25,1.60)$ & $1.78(1.47,2.15)$ & $1.10(0.94,1.31)$ & $1.20(1.04,1.38)$ \\
\hline Diabetes & $1.53(1.30,1.79)$ & - & $1.46(1.18,1.80)$ & $1.64(1.38,1.96)$ \\
\hline Cause of kidney failure & & - & & \\
\hline Glomerulonephritis & $0.70(0.60,0.82)$ & & $0.67(0.55,0.82)$ & $0.67(0.56,0.80)$ \\
\hline Diabetes & $0.89(0.72,1.12)$ & & $1.14(0.85,1.52)$ & $1.07(0.84,1.38)$ \\
\hline Hypertension/renovascular disease & 1.00 & & 1.00 & 1.00 \\
\hline Cystic & $0.62(0.52,0.75)$ & & $0.79(0.64,0.99)$ & $0.72(0.59,0.89)$ \\
\hline Analgesic nephropathy & $1.68(1.18,2.39)$ & & $1.53(1.04,2.24)$ & $1.48(1.03,2.12)$ \\
\hline Others & $0.77(0.66,0.91)$ & & $0.85(0.68,1.07)$ & $0.89(0.73,1.09)$ \\
\hline Dialysis duration (in years) & $1.06(1.05,1.08)$ & - & $1.09(1.07,1.11)$ & $1.09(1.07,1.11)$ \\
\hline \multicolumn{5}{|l|}{ Transplant factors } \\
\hline \multicolumn{5}{|l|}{ HLA-DR mismatches } \\
\hline 0 & 1.00 & 1.00 & 1.00 & 1.00 \\
\hline 1 & $1.26(1.14,1.38)$ & $1.59(1.39,1.82)$ & $0.95(0.84,1.09)$ & $1.04(0.92,1.17)$ \\
\hline 2 & $1.27(1.14,1.41)$ & $1.65(1.43,1.92)$ & $1.03(0.89,1.19)$ & $1.15(1.02,1.30)$ \\
\hline Peak PRA (\%) & - & & - & - \\
\hline $0-10$ & & 1.00 & & \\
\hline $11-50$ & & $1.24(1.06,1.44)$ & & \\
\hline $51-80$ & & $1.31(1.02,1.68)$ & & \\
\hline$>80$ & & $1.61(1.26,2.06)$ & & \\
\hline \multicolumn{5}{|l|}{ Transplant era } \\
\hline 2000-2004 & 1.00 & 1.00 & 1.00 & 1.00 \\
\hline 2005-2008 & 1.07 (0.97, 1.18) & $1.21(1.06,1.38)$ & $0.90(0.78,1.04)$ & $0.92(0.82,1.04)$ \\
\hline 2009-2012 & $1.06(0.94,1.18)$ & $1.24(1.06,1.46)$ & $0.85(0.72,0.99)$ & $0.90(0.78,1.04)$ \\
\hline $2013-2017$ & $1.02(0.87,1.19)$ & $1.45(1.16,1.81)$ & $0.68(0.54,0.85)$ & $0.82(0.67,1.01)$ \\
\hline
\end{tabular}

Data presented as adjusted hazard ratio (HR) and 95\% confidence intervals (95\% Cl) in the multi-variable adjusted Cox regression models, with the estimates of the covariates selected by group least absolute shrinkage and selection operator (LASSO) shown. eGFR, estimated glomerular filtration rate; PRA, panel reactive antibody; HLA-human leukocyte antigen.

There are several strengths and limitations in this study. The prospective nature of a contemporary cohort of kidney transplant recipients and the near completeness of the available data suggest that ascertainment biases of the exposure and outcome measures were minimized and that the study findings reflect current clinical practice. Indication bias remained a possibility because there may have been systematic differences in how clinicians manage kidney transplant recipients with differing eGFR values at 12 -months post-transplant. However, the direction of this bias is likely towards the null hypothesis because people with lower eGFR may receive closer monitoring or changes to the management approach due to the lower eGFR. Even though there were multiple confounding factors adjusted for in the analyses, there are likely to be several unmeasured and residual confounders. These include the overall exposure and utilization of immunosuppression (according to clinical risk), the impact of various adverse clinical events/hospitalizations occurring during the time course of the follow-up period, lack of availability of biopsy data and changing nature of immunological risk (such as evidence of transplant glomerulopathy, presence of interstitial fibrosis/tubular atrophy, development of de novo donor-specific anti-HLA antibody), presence of and severity of proteinuria and the development (and severity) of de novo comorbid conditions such as post-transplant diabetes and hypertension that may have influenced allograft function and allograft survival post-transplant; which were not adequately collected by the ANZDATA registry but may potentially have modified our study findings. It was determined $a$ priori that change in eGFR would not be considered in this study given that the majority of landmark clinical studies had utilized a single time point eGFR measurement as the primary or secondary endpoint. However, our other work has shown that change in eGFR 

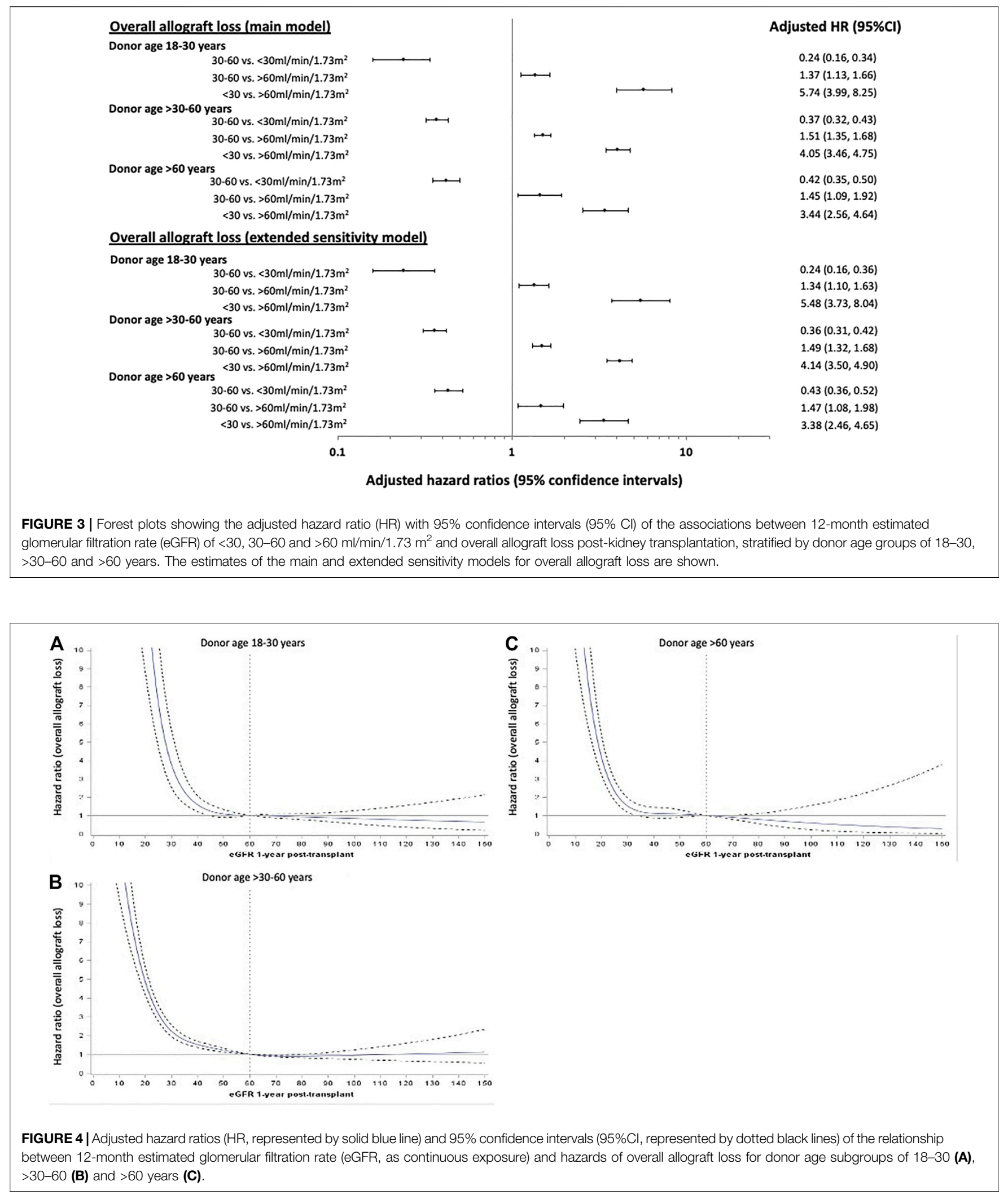
is a valuable predictor of long-term outcomes (19). Misclassification bias of actual allograft function may have occurred, however, measured GFR was impractical and costly in the real-world setting. In addition, given the small number of kidney transplant recipients of younger deceased donor kidneys that achieved 12month eGFR values of $<30 \mathrm{ml} / \mathrm{min} / 1.73 \mathrm{~m}^{2}$, there is likely considerable uncertainty in the estimates to provide an accurate assessment of the true difference between eGFR and allograft outcomes for this group.

In conclusions, our study shows that the association between 12-month eGFR and allograft outcome is modified by donor age. Even though the relationship between eGFR and allograft outcome is similar among different donor age subgroups, an identical single timepoint eGFR as a prognostic indicator of allograft survival and the attainment of a range of eGFR thresholds varies according to these subgroups.

\section{CAPSULE SENTENCE SUMMARY}

Reduced estimated glomerular filtration rate (eGFR) 12-month post-transplant is associated with adverse long-term allograft outcomes but whether donor factors such as age, modify this association in unknown. Using data from the Australia and New Zealand Dialysis and Transplant (ANZDATA) registry, we have shown that the relationship between 12-month eGFR and allograft loss was modified by donor age. Even though the trend and nature of the relationships between 12-month eGFR and allograft loss were similar, the magnitudes of the risk were dissimilar among donor age subgroups.

\section{DATA AVAILABILITY STATEMENT}

The data analyzed in this study is subject to the following licenses/ restrictions: Use of deidentified data can be requested from ANZDATA registry. Requests to access these datasets should be directed to requests@anzdata.org.au.

\section{ETHICS STATEMENT}

The studies involving human participants were reviewed and approved by University of Western Australia Human Research

\section{REFERENCES}

1. Go AS, Chertow GM, Fan D, McCulloch CE, Hsu CY. Chronic Kidney Disease and the Risks of Death, Cardiovascular Events, and Hospitalization. N Engl J Med (2004) 351(13):1296-305. doi:10.1056/ nejmoa041031

2. Lewis JR, Lim W, Dhaliwal SS, Zhu K, Lim EM, Thompson PL, et al. Estimated Glomerular Filtration Rate as an Independent Predictor of Atherosclerotic Vascular Disease in Older Women. BMC Nephrol (2012) 13:58. doi:10.1186/ 1471-2369-13-58

3. Chronic Kidney Disease Prognosis C, Matsushita K, van der Velde M, Astor BC, Woodward M, Levey AS, et al. Association of Estimated Glomerular
Ethics Committee. Written informed consent for participation was not required for this study in accordance with the national legislation and the institutional requirements.

\section{AUTHOR CONTRIBUTIONS}

$\mathrm{WL}, \mathrm{EO}$, and GW conceived the proposal WL, EO, and GW analysed the data. All authors contributed to the writing of the manuscript.

\section{CONFLICT OF INTEREST}

The authors declare that the research was conducted in the absence of any commercial or financial relationships that could be construed as a potential conflict of interest.

\section{ACKNOWLEDGMENTS}

The authors would like to gratefully acknowledge the substantial contributions of the entire Australian and New Zealand nephrology community (physicians, surgeons, database managers, nurses, renal operators and patients) that provide information to, and maintain, the ANZDATA database. The data reported here have been supplied by the ANZDATA registry. The interpretation and reporting of these data are the authors' responsibility and in no way should be seen as official policy or interpretation of the ANZDATA registry. DJ is supported by a National Health and Medical Research Council Practitioner Fellowship. GW is supported by a National Health and Medical Research Council Career Development Fellowship. NL is supported by a Clinician Research Fellowship from the Department of Health and Raine Medical Research Foundation. EO is supported by a Heart Foundation Future Leader Fellowship (Award ID: 102538).

\section{SUPPLEMENTARY MATERIAL}

The Supplementary Material for this article can be found online at: https://www.frontierspartnerships.org/articles/10.3389/ti.2022. 10199/full\#supplementary-material

Filtration Rate and Albuminuria with All-Cause and Cardiovascular Mortality in General Population Cohorts: a Collaborative Meta-Analysis. Lancet (2010) 375(9731):2073-81. doi:10.1016/S0140-6736(10)60674-5

4. O’Hare AM, Bertenthal D, Covinsky KE, Landefeld CS, Sen S, Mehta K, et al. Mortality Risk Stratification in Chronic Kidney Disease: One Size for All Ages? J Am Soc Nephrol (2006) 17(3):846-53. doi:10.1681/ASN. 2005090986

5. Kasiske BL, Israni AK, Snyder JJ, Skeans MA. The Relationship between Kidney Function and Long-Term Graft Survival after Kidney Transplant. Am J Kidney Dis (2011) 57(3):466-75. doi:10.1053/j.ajkd.2010.10.054

6. Ibrahim A, Garg AX, Knoll GA, Akbari A, White CA. Kidney Function Endpoints in Kidney Transplant Trials: a Struggle for Power. Am J Transplant (2013) 13(3):707-13. doi:10.1111/ajt.12050 
7. White CA, Siegal D, Akbari A, Knoll GA. Use of Kidney Function End Points in Kidney Transplant Trials: a Systematic Review. Am J Kidney Dis (2010) 56(6):1140-57. doi:10.1053/j.ajkd.2010.08.015

8. Hariharan S, McBride MA, Cherikh WS, Tolleris CB, Bresnahan BA, Johnson CP. Post-transplant Renal Function in the First Year Predicts Long-Term Kidney Transplant Survival. Kidney Int (2002) 62(1):311-8. doi:10.1046/j. 1523-1755.2002.00424.x

9. Schnitzler MA, Lentine KL, Axelrod D, Gheorghian A, You M, Kalsekar A, et al. Use of 12-Month Renal Function and Baseline Clinical Factors to Predict Long-Term Graft Survival. Transplantation (2012) 93(2):172-81. doi:10.1097/ tp.0b013e31823ec02a

10. He X, Moore J, Shabir S, Little MA, Cockwell P, Ball S, et al. Comparison of the Predictive Performance of eGFR Formulae for Mortality and Graft Failure in Renal Transplant Recipients. Transplantation (2009) 87(3):384-92. doi:10. 1097/tp.0b013e31819004a1

11. Pascual J, Berger SP, Witzke O, Tedesco H, Mulgaonkar S, Qazi Y, et al. Everolimus with Reduced Calcineurin Inhibitor Exposure in Renal Transplantation. Jasn (2018) 29(7):1979-91. doi:10.1681/asn. 2018010009

12. Ekberg H, Tedesco-Silva H, Demirbas A, Vítko Š, Nashan B, Gürkan A, et al. Reduced Exposure to Calcineurin Inhibitors in Renal Transplantation. N Engl J Med (2007) 357(25):2562-75. doi:10.1056/nejmoa067411

13. Lebranchu Y, Thierry A, Toupance O, Westeel PF, Etienne I, Thervet E, et al. Efficacy on Renal Function of Early Conversion from Cyclosporine to Sirolimus 3 Months after Renal Transplantation: Concept Study. Am J Transplant : official J Am Soc Transplant Am Soc Transpl Surgeons (2009) 9(5):1115-23. doi:10.1111/j.1600-6143.2009.02615.x

14. Tong A, Craig JC, Nagler EV, Van Biesen W. Composing a New Song for Trials: the Standardized Outcomes in Nephrology (SONG) Initiative. Nephrol Dial Transpl (2017) 32(12):1963-6. doi:10.1093/ndt/gfx288

15. Sautenet B, Tong A, Manera KE, Chapman JR, Warrens AN, Rosenbloom D, et al. Developing Consensus-Based Priority Outcome Domains for Trials in Kidney Transplantation. Transplantation (2017) 101(8):1875-86. doi:10.1097/ tp.0000000000001776

16. Clayton PA, Dansie K, Sypek MP, White S, Chadban S, Kanellis J, et al. External Validation of the US and UK Kidney Donor Risk Indices for Deceased Donor Kidney Transplant Survival in the Australian and New Zealand Population. Nephrology, Dialysis, Transplantationofficial Publication Eur Dial Transpl Assoc - Eur Ren Assoc (2019) 34(12):2127-31. doi:10.1093/ ndt/gfz090

17. Rao PS, Schaubel DE, Guidinger MK, Andreoni KA, Wolfe RA, Merion RM, et al. A Comprehensive Risk Quantification Score for Deceased Donor Kidneys: the Kidney Donor Risk index. Transplantation (2009) 88(2): 231-6. doi:10.1097/tp.0b013e3181ac620b

18. Rao PS, Ojo A. The Alphabet Soup of Kidney Transplantation: SCD, DCD, ECD-Fundamentals for the Practicing Nephrologist. Cjasn (2009) 4(11): 1827-31. doi:10.2215/cjn.02270409

19. Clayton PA, Lim WH, Wong G, Chadban SJ. Relationship between eGFR Decline and Hard Outcomes after Kidney Transplants. Jasn (2016) 27(11): 3440-6. doi:10.1681/asn.2015050524
20. Lenihan CR, O'Kelly P, Mohan P, Little D, Walshe JJ, Kieran NE, et al. MDRD-estimated GFR at One Year post-renal Transplant Is a Predictor of Long-Term Graft Function. Ren Fail (2008) 30(4):345-52. doi:10.1080/ 08860220801947686

21. Elm Ev., Altman DG, Egger M, Pocock SJ, Gøtzsche PC, Vandenbroucke JP. Strengthening the Reporting of Observational Studies in Epidemiology (STROBE) Statement: Guidelines for Reporting Observational Studies. BMJ (2007) 335(7624):806-8. doi:10.1136/bmj. 39335.541782.ad

22. Howell M, Tong A, Wong G, Craig JC, Howard K. Important Outcomes for Kidney Transplant Recipients: a Nominal Group and Qualitative Study. Am J Kidney Dis (2012) 60(2):186-96. doi:10.1053/j.ajkd.2012.02.339

23. Vincenti F, Charpentier B, Vanrenterghem Y, Rostaing L, Bresnahan B, Darji P, et al. A Phase III Study of Belatacept-Based Immunosuppression Regimens versus Cyclosporine in Renal Transplant Recipients (BENEFIT Study). Am J Transplant : official J Am Soc Transplant Am Soc Transpl Surgeons (2010) 10(3):535-46. doi:10.1111/j.1600-6143.2009.03005.x

24. Vincenti F, Rostaing L, Grinyo J, Rice K, Steinberg S, Gaite L, et al. Belatacept and Long-Term Outcomes in Kidney Transplantation. N Engl J Med (2016) 374(4):333-43. doi:10.1056/nejmoa1506027

25. Levey AS, Stevens LA, Schmid CH, Zhang Y, Castro AF, Feldman HI, et al. A New Equation to Estimate Glomerular Filtration Rate. Ann Intern Med (2009) 150(9):604-12. doi:10.7326/0003-4819-150-9-200905050-00006

26. Gaines BR, Kim J, Zhou H. Algorithms for Fitting the Constrained Lasso. J Comput Graphical Stat (2018) 27(4):861-71. doi:10.1080/10618600.2018.1473777

27. Merion RM, Ashby VB, Wolfe RA, Distant DA, Hulbert-Shearon TE, Metzger RA, et al. Deceased-donor Characteristics and the Survival Benefit of Kidney Transplantation. JAMA (2005) 294(21):2726-33. doi:10.1001/jama.294.21. 2726

28. Lim WH, Clayton P, Wong G, Campbell SB, Cohney S, Russ GR, et al. Outcomes of Kidney Transplantation from Older Living Donors. Transplantation (2013) 95(1):106-13. doi:10.1097/tp. 0b013e318277b2be

29. Gill J, Bunnapradist S, Danovitch GM, Gjertson D, Gill JS, Cecka M. Outcomes of Kidney Transplantation from Older Living Donors to Older Recipients. Am J Kidney Dis (2008) 52(3):541-52. doi:10.1053/j.ajkd.2008. 05.017

30. Mengel M, Chapman JR, Cosio FG, Cavaillé-Coll MW, Haller H, Halloran PF, et al. Protocol Biopsies in Renal Transplantation: Insights into Patient Management and Pathogenesis. Am J Transpl (2007) 7(3):512-7. doi:10. $1111 /$ j.1600-6143.2006.01677.x

Copyright $\odot 2022$ Lim, Ooi, Pilmore, Johnson, McDonald, Clayton, Hawley, Mulley, Francis, Collins, Jaques, Larkins, Davies, Wyburn, Chadban and Wong. This is an open-access article distributed under the terms of the Creative Commons Attribution License (CC BY). The use, distribution or reproduction in other forums is permitted, provided the original author(s) and the copyright owner(s) are credited and that the original publication in this journal is cited, in accordance with accepted academic practice. No use, distribution or reproduction is permitted which does not comply with these terms. 